\title{
Interaction of Metals with Suspended Graphene Observed by Transmission Electron Microscopy
}

${ }_{3}$ Recep Zan, ${ }^{\dagger \neq}$ Ursel Bangert, ${ }^{*}{ }^{\ddagger}$ Quentin Ramasse, ${ }^{\S}$ and Konstantin S. Novoselov ${ }^{\dagger}$

$4{ }^{\dagger}$ School of Physics and Astronomy and ${ }^{\ddagger}$ School of Materials, The University of Manchester, Manchester, M13 9PL, United Kingdom

$5{ }^{\S}$ SuperSTEM Laboratory, STFC Daresbury, Daresbury WA4 4AD, United Kingdom

6 ABSTRACT: In this Perspective, we present an overview of how different metals interface with suspended graphene, providing a closer look into the metal-graphene interaction by employing high-resolution transmission electron microscopy, especially using high-angle dark field imaging. All studied metals favor sites on the omnipresent hydrocarbon surface contamination rather than on the clean graphene surface and present nonuniform distributions, which never result in continuous films but instead in clusters or nanocrystals, indicating a weak interaction between the metal and graphene. This behavior can be altered to some degree by surface pretreatment (hydrogenation) and high-temperature vacuum annealing. Graphene etching is observed in a scanning transmission electron microscope (STEM) under high vacuum and $60 \mathrm{kV}$ electron beam acceleration voltage conditions for all metals, except for Au. This unusual metal-mediated etching sheds new light on the metal-graphene interaction; it might explain the observed higher frequency of cluster nucleation for certain transition metals and might have implications regarding controlled nanomanipulation, that is, for self-assembly and sculpturing of future graphene-based devices.

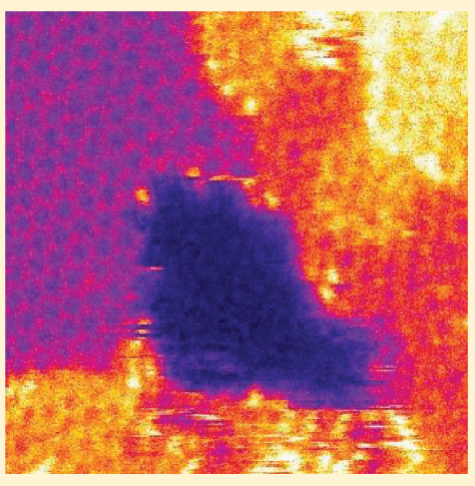

$20 \bigcirc$ raphene, the first two-dimensional material to be isolated, ${ }_{21}-$ has become the focus of intense fundamental research 22 due to its extraordinary properties, but even more so, it has 23 spurred massive interest from various fields into studies 24 regarding nanotechnology applications. ${ }^{1,2}$ An area of immense 25 importance in all of this is the study of the metal-graphene 26 interaction because metals have to be used in every single appli27 cation of graphene as a functional material. ${ }^{1,3,4}$ Metal effects on 28 transport, electronic, magnetic, and structural properties of 29 graphene have been investigated both experimentally ${ }^{3,4}$ and 30 theoretically, ${ }^{5,6}$ by means of density functional theory (DFT) 31 with more emphasis on theoretical than on experimental studies.

Due to its large surface area, chemical stability, and low cost, graphene is a highly desirable support for metal catalysts. However, due to the chemical inertness of graphene, there are two barriers to overcome, which affect the metal-graphene interaction, the stabilization of nanoparticles, and achievement of uniform distributions.
Due to its large surface area, chemical stability, and low cost, $32 \mathrm{p}$ graphene is a highly desirable support for metal catalysts. How- 33 ever, due to the chemical inertness of graphene, there are two 34 barriers to overcome, which affect the metal-graphene inter- 35 action, the stabilization of nanoparticles, and achievement of 36 uniform distributions. Different methods have been suggested to 37 deal with these issues. Introducing vacancies ${ }^{6,7}$ and applying 38 strain $^{8,9}$ in the graphene sheet are ways of stabilizing metal clusters. 39 Vacancies behave like traps for metal atoms and clusters due to the 40 presence of dangling bonds, thus increasing the reactivity of 41 graphene. ${ }^{10}$ The other method consists of functionalizing graphene 42 in solution (graphene oxidation), which also allows one to obtain 43 chemically processable graphene. ${ }^{11-15}$ During chemical function- 44 alization, oxy-functional groups are introduced, which act as 45 nucleation sites and facilitate seeding and growth of metal nano- 46 clusters. However, it should be noted that the metal behavior on 47 modified graphene is governed by the chemical method used and 48 can therefore vary.

Graphene has furthermore been used as an ideal transparent 50 support for transmission electron microscopy (TEM) studies 51 directly focused on nanoparticles (i.e., gold ${ }^{16}$ and cobalt ${ }^{17}$ ). $\quad 52$

Metals on graphene were found to induce a large enhance- 53 ment of the Raman signal. ${ }^{18}$ A correlation has been recently 54 found between the enhancement factor and the G band splitt- 55 ing for a different number of layers. ${ }^{19}$

Several scanning tunnelling microscopy (STM) studies have 57 been performed to investigate electrical and structural properties of 58

Received: December 15, 2011

Accepted: March 8, 2012 

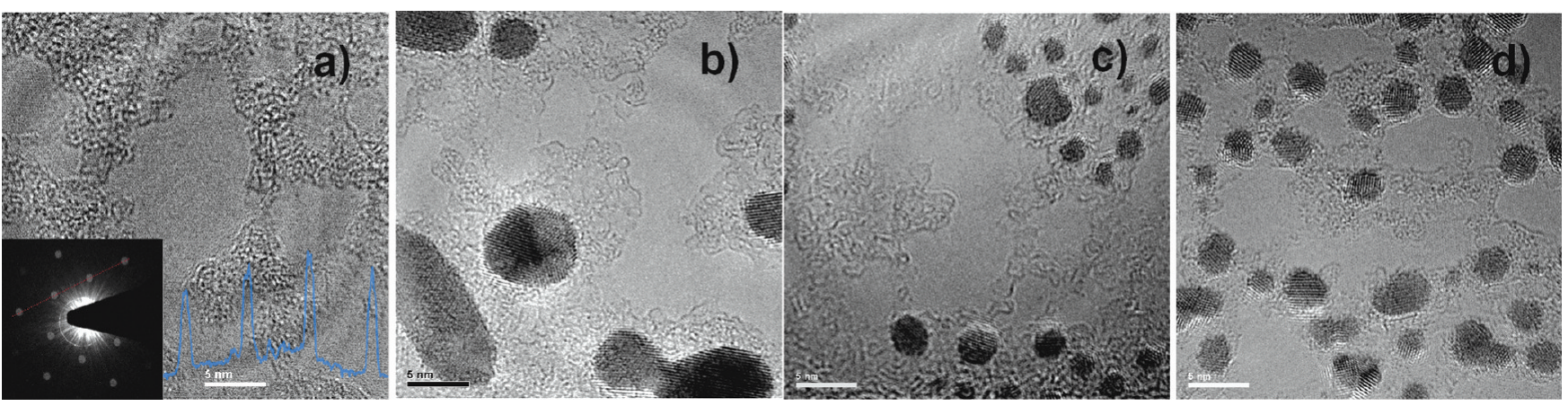

Figure 1. (a) Bright field (BF) image of pristine monolayer graphene. The inset shows the diffraction pattern and intensity profile along the red line in the diffraction pattern. BF image of 2 Å gold evaporated on (b) pristine, (c) two-cycle-hydrogenated, and (d) four-cycle hydrogenated monolayer graphene. The scale $(5 \mathrm{~nm})$ is chosen to be same in all images for accurate comparison.

59 cluster arrays on graphene. However, all reported STM studies are 60 performed on graphene on a substrate; therefore, substrate effects 61 have to be taken into consideration, in particular, when compared 62 to TEM studies, which are conducted on suspended graphene. ${ }^{20-22}$ 63 Metals are also used for graphene tailoring, that is, for cutting 64 graphene sheets into nanoribbons. ${ }^{23-25}$ However, the respec65 tive experiments are mainly conducted at elevated temperature 66 in gas environments and do not present a controllable way of 67 slicing graphene yet. Metal graphene composites are furthermore 68 used for practical applications, for example, in transistors, ${ }^{26}$ electro69 chemical catalysis, ${ }^{12}$ biosensors, ${ }^{27}$ solar cells, ${ }^{28}$ and batteries. ${ }^{29}$

70 However, there is a lack of electron microscopy studies, in parti71 cular, of high-resolution TEM, and this is limiting the under72 standing of the metal-graphene interaction. In this Perspective, we 73 present an overview of stationary mode TEM and scanning mode 74 TEM, that is, STEM, of metal-graphene interfaces, providing a 75 closer look into the metal-graphene interaction.

76 Regardless of the production method, whether produced by 77 exfoliation $^{1}$ or CVD growth, ${ }^{30}$ suspended pristine graphene is 78 known to react strongly with hydrocarbons. These most 79 probably arise from air exposure and/or remnants of adhesives 80 used during extraction, transfer, and handling of graphene 81 (Figure 1a). Although the microscope column has a relatively 82 high vacuum $\left(\sim 10^{-8}\right.$ Torr $)$, traces of $\mathrm{CO}, \mathrm{CO}_{2}$, and $\mathrm{H}_{2} \mathrm{O}$ can 83 be present inside of the instrument itself. Clean graphene areas 84 (free from residue) vary in size from a few $\mathrm{nm}^{2}$ to a few hundred $85 \mathrm{~nm}^{2}$; these areas are surrounded by worm-like hydrocarbon 86 contamination (Figure 1a). Prior to metal deposition (in our 87 case, via evaporation) onto the graphene and consecutive 88 (S)TEM investigations, the number of graphene layers was 89 identified. The most convenient method to do this is via 90 electron diffraction by comparing first- and second-order dif91 fraction spot intensities (inset Figure 1a). ${ }^{31}$ To begin with, one 92 of the technologically important metals, Au, has been studied. 93 Gold atoms and clusters are mainly observed on hydrocarbon 94 contamination, as previously reported. ${ }^{32,33}$ The cluster sizes 95 vary from about 1 to $5 \mathrm{~nm}$ in diameter (Figure $1 \mathrm{~b}$ ), and the 96 clusters are not equally distributed on the graphene surface 97 (Figure 1b). As a result of surface treatment, in our case, by 98 exposing pristine graphene samples to a cold hydrogen plasma ${ }^{34}$ 99 for one, two $(\sim 30 \mathrm{~min})$, and four cycles $(\sim 60 \mathrm{~min})$, the cluster 100 distributions and sizes are affected, although clusters remain on 101 hydrocarbon contamination. ${ }^{35}$ Gold cluster distributions become 102 more uniform in hydrogenated samples (Figure $1 \mathrm{c}$ and $\mathrm{d}$ ), and 103 cluster sizes become similar, in particular, after four-cycle hydro104 genation. Coalescence of gold clusters is observed for both 105 pristine and hydrogenated samples as a result of long electron beam exposure. However, it is much more pronounced in 106 hydrogenated samples. Coalescence is observed within a few 107 seconds on hydrogenated samples, whereas it takes longer (50 s) 108 in pristine samples.

\section{Gold cluster distributions become more uniform in hydrogenated samples, and cluster sizes become similar.}

Another way to study metal clusters on graphene is to anneal 110 them either in a gas environment or in vacuum at elevated 111 temperature. In situ annealing and imaging in the microscope in 112 high vacuum is a feasible way to investigate gold cluster stability 113 at high temperatures. As previously observed by our group, 114 annealing pristine samples at $\sim 700{ }^{\circ} \mathrm{C}$ in high vacuum is 115 sufficient to eliminate most of the hydrocarbon contamination 116 from the graphene surface. As can be seen in Figure 2a, as the 117 clean graphene areas increase due to evaporation of hydro- 118 carbon contamination during high-vacuum annealing, the gold 119 clusters, which reside in the hydrocarbons, are forced to move 120 toward each other. However, coalescence has not been 121 observed yet at this temperature (Figure 2a and b). As a next 122 step, the annealing temperature was increased to $950{ }^{\circ} \mathrm{C}$, where 123 gold clusters agglomerated, almost melted, and, as a result, have 124 flattened, and no contamination was observed (Figure 2c). Lastly, 125 for comparison, few-layer graphene with the same amount of gold 126 was annealed at $700{ }^{\circ} \mathrm{C}$ in high vacuum (Figure 2d). It was found 127 that gold cluster sizes became bigger, and their distributions were 128 less uniform than on monolayer graphene, resulting in much more 129 open space, free from residue, on the graphene surface. 130

Gold clusters have been observed to react more strongly with 131 few-layer than with monolayer graphene, either via lattice 132 defects or a very thin interlayer of hydrocarbon contamination. 133 Due to the mismatch between the gold and graphene lattice, 134 rotational Moiré effects can be observed directly in lattice 135 images and also by two rotated sets of diffraction spots in the 136 Fourier transform (FFT) of these images (Figure 3a). Moiré 137 effects have not been observed for boron nitride (BN) with 138 similar amounts of gold evaporation (Figure 3b). As can be 139 seen from the inset in Figure $3 \mathrm{~b}, \mathrm{BN}$ and gold diffraction spots 140 coincide. Au clusters on BN also appear to sit exclusively on 141 hydrocarbon layers. The slightly stronger interaction between 142 

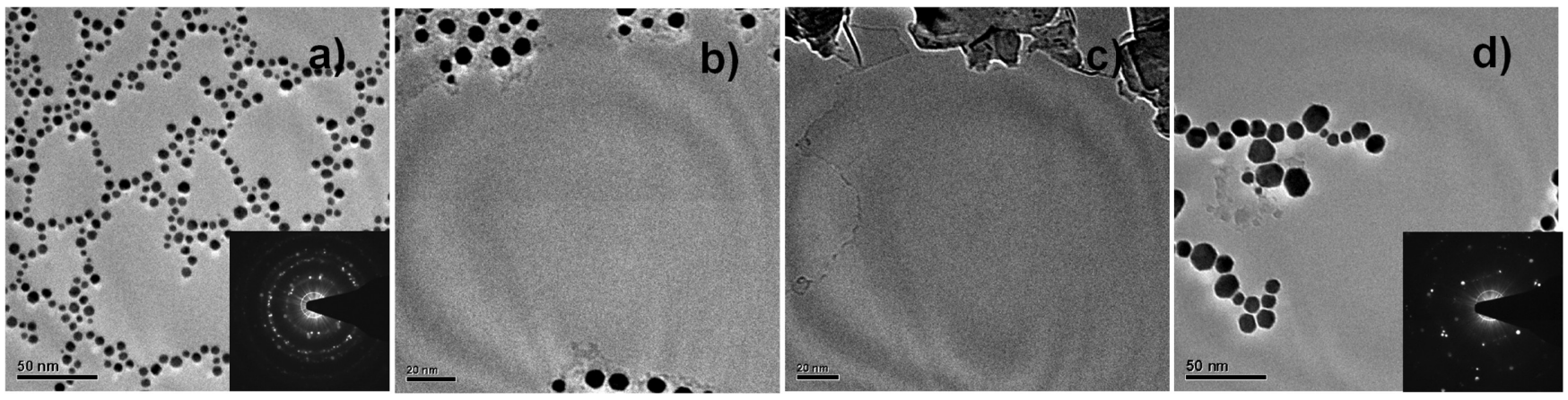

Figure 2. BF images of $2 \AA$ gold (a) evaporated onto monolayer graphene and annealed at $700{ }^{\circ} \mathrm{C}$ with the diffraction pattern as the inset, (b) showing a magnified image of (a), (c) as (a) but annealed at $950{ }^{\circ} \mathrm{C}$, and (d) on few-layer graphene and annealed at $700{ }^{\circ} \mathrm{C}$ with the diffraction pattern as the inset. The scale bar is the same in (a) and (d), $50 \mathrm{~nm}$, and it is similar in (b) and (c), $20 \mathrm{~nm}$.
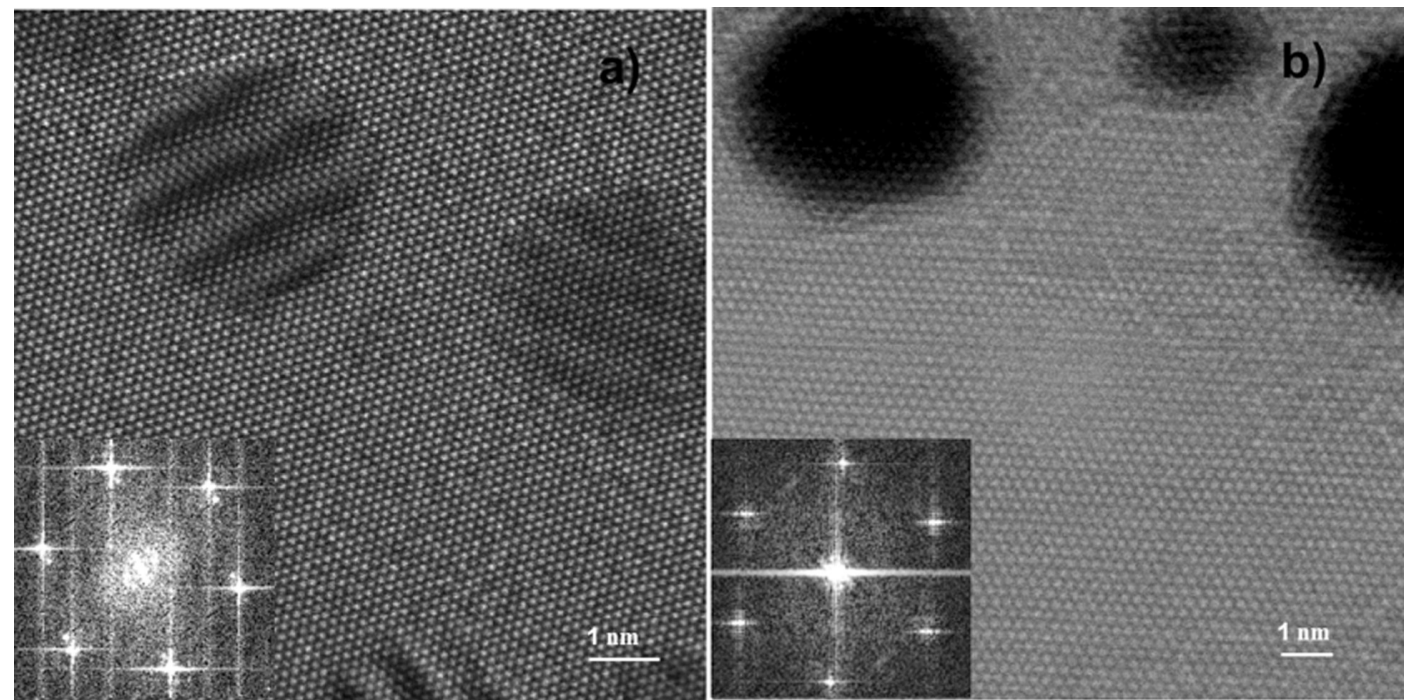

Figure 3. BF images of 2 Å gold evaporated onto few-layer (a) graphene and (b) boron nitride. The corresponding FFTs are shown as insets. The scale bar is the same in (a) and (b), $1 \mathrm{~nm}$.

143 gold and few-layer graphene in this experiment might be 144 attributed to the higher number $(>5)$ of graphene sheets with 145 subsurface layers making a significant contribution to the 146 bonding, whereas the BN flake was thinner ( $<5$ layers).

147 Gold has never been observed to introduce any damage into 148 graphene; this conclusion can be drawn with high certainty 149 from STEM studies, ${ }^{33}$ where a $60 \mathrm{kV}$ acceleration voltage has 150 been used for imaging, an energy which is known to be well 151 below the displacement threshold for graphene. ${ }^{36}$ In contrast, 152 damaging of graphene has been observed in the presence of $\mathrm{Al}$, $153 \mathrm{Ti}, \mathrm{Cr}, \mathrm{Pd}$, and $\mathrm{Ni}$, although their interaction with graphene 154 varies; for example, $\mathrm{Al}, \mathrm{Cr}$, and $\mathrm{Ti}$ are much more reactive than $155 \mathrm{Pd}$ and $\mathrm{Ni}$. Except for a few rare instances, clusters of all of 156 these metals are found to reside on hydrocarbon chains, as for 157 the case of Au. However, observation during repeated STEM 158 scans shows that smaller clusters and individual atoms are 159 drawn out of their initial positions, that is, from the middle of 160 contamination patches to the edge of the contamination. As 161 soon as metals reach the border between the hydrocarbon and 162 clean graphene, they interact with the clean graphene surface. 163 Initially, point defects (vacancies) are created, and this pro164 cess repeats itself as long as new metal atoms are supplied to 165 the emerging vacancy clusters from nearby metal clusters. 166 Atomic-resolution high-angle annular dark field (HAADF)-
STEM imaging has been employed to study individual 167 adatoms on graphene. The scattering probability here follows 168 an approximate $Z^{2}$ law, where $Z$ is the atomic number, which 169 makes single-atom impurity detection (especially of impurities 170 heavier than carbon) possible, and the interpretation of the 171 images is rather straightforward.

$172 \mathrm{p}$

\section{Damaging of graphene has been observed in the presence of $\mathrm{Al}$, $\mathrm{Ti}, \mathrm{Cr}, \mathrm{Pd}$, and $\mathrm{Ni}$, although their interaction with graphene varies.}

The etching process is shown for $\mathrm{Al}$ in the HAADF images in 173 Figure 4. Figure 4a shows a clean, intact graphene patch (black) 174 surrounded by hydrocarbons (gray) with Al clusters (white). 175 Various stages of hole formation are shown in Figure $4 b-d$, and 176 Figure $4 \mathrm{e}$ shows the hole after etching has more or less ceased. 177 In Figure $4 \mathrm{~b}-\mathrm{d}$, the hole is decorated by newly arriving $\mathrm{Al} 178$ atoms, leading to enlargement, whereas no such atoms can be 179 

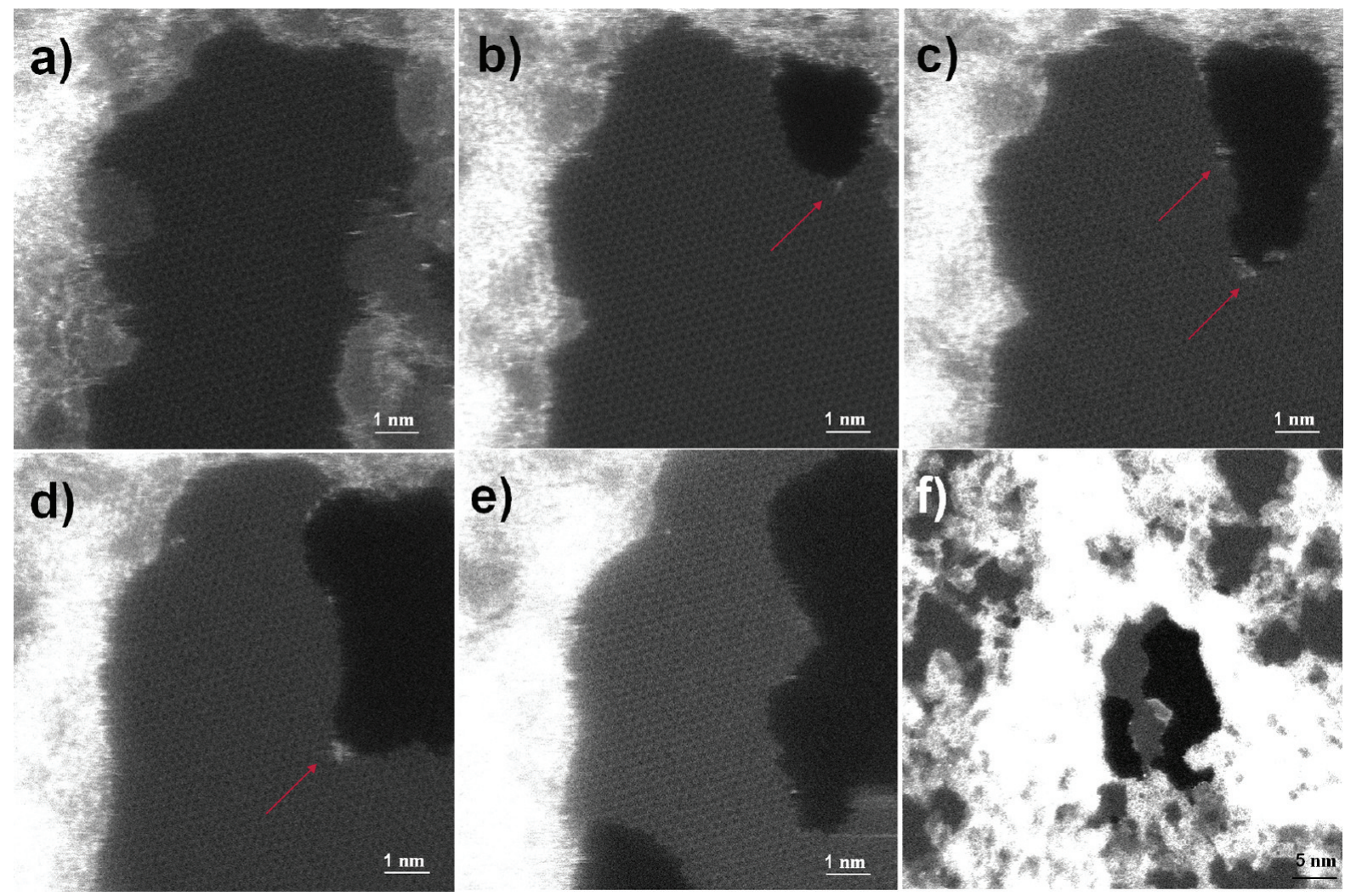

Figure 4. HAADF images of graphene etching in the presence of an aluminum layer of 2 A nominal thickness (a) before etching, (b) after the start of the hole formation, (c) after hole enlargement in subsequent scans, (d) after continued etching as a result of a sustained supply of $\mathrm{Al}$ atoms to the hole's edge (some $\mathrm{Al}$ atoms are indicated by red arrows in $(\mathrm{b}-\mathrm{d})$, and $(\mathrm{e})$ after the etching process has almost stopped because the $\mathrm{Al}$ atom supply has ceased. (f) A lower magnification overview of the $\mathrm{Al}$ distribution and hole evolution. The scale bar is the same in (a-e), $1 \mathrm{~nm}$.
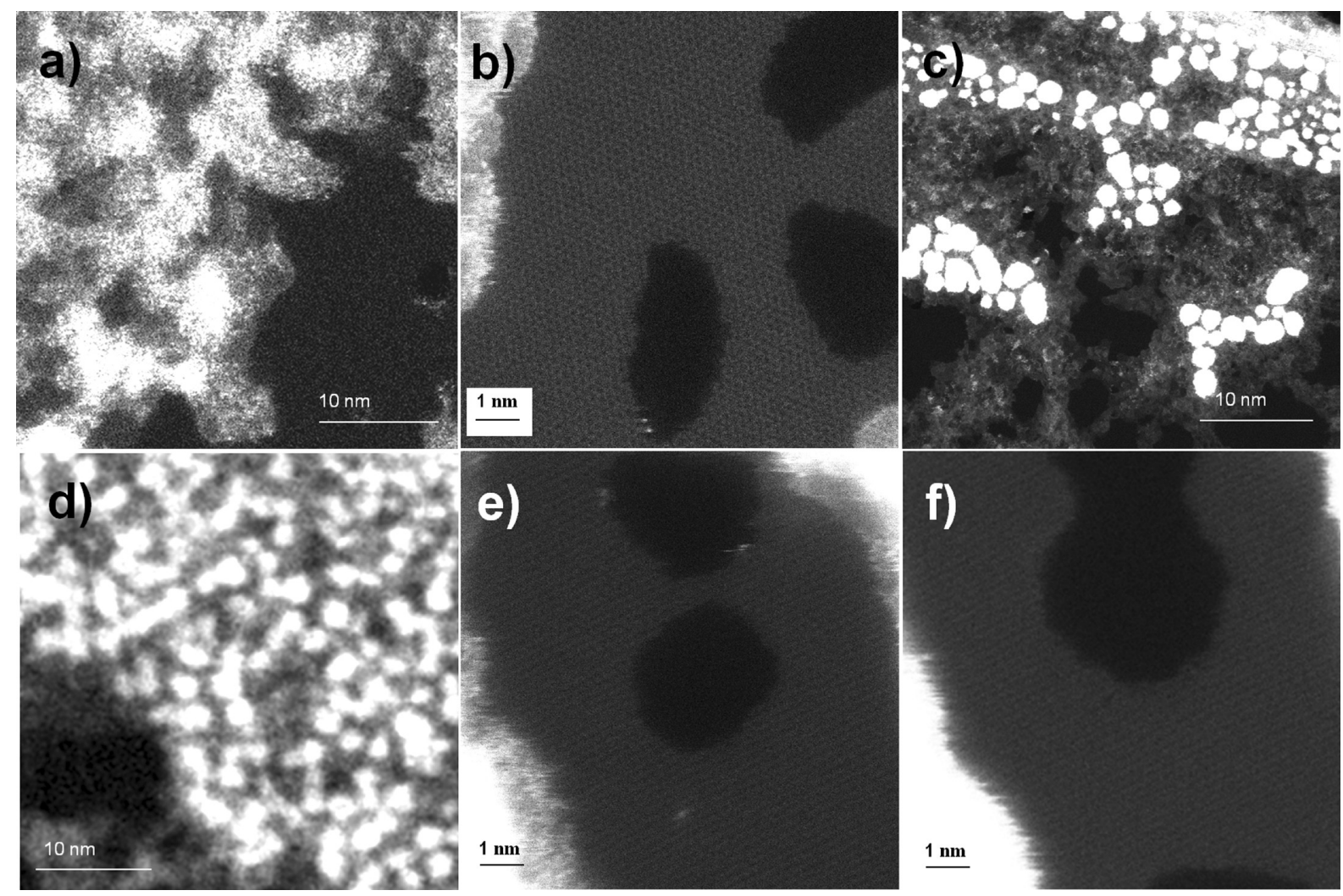

Figure 5. (a) HAADF image (overview) of $2 \AA$ titanium evaporated onto monolayer graphene, (b) a magnified image showing direct etching of the basal plane as a result of the strong interaction between Ti and graphene, ( $\mathrm{c}$ and d) overview of Pd and Cr distributions on graphene, (e) magnified image showing hole initiation due to $\mathrm{Cr}$ at the border of hydrocarbon contamination as well as directly on the basal plane, and (f) coalescence of the holes in (e) after repeated scans. 
180 observed in Figure 4e. An overview at smaller magnification of 181 an intermediate etching stage together with the aluminum 182 distribution is shown in Figure 4f. Figure 4 demonstrates clearly 183 that the etching progresses from the border of the contamina184 tion into clean graphene as long as metal atoms are present at 185 the hole; these appear to mediate the etching. In the absence of 186 metal atoms at the hole, no such progression of the etching is 187 observed.

188 This destructive behavior has been predicted by recent DFT 189 calculations of $\mathrm{Ni}, \mathrm{Al}, \mathrm{Co}$, and $\mathrm{Fe}$ on graphene; these elements 190 lower the vacancy formation energy in graphene. ${ }^{37}$ The same 191 calculations for $\mathrm{Au}$ on graphene do not predict such behavior 192 because vacancy formation energies in this case were found to 193 be similar to those of pristine graphene. However, catalytic 194 oxidation $^{24}$ or hydrogenation $^{26}$ of carbon atoms in the presence 195 of metal nanoparticles in graphene could be proposed as an 196 alternative mechanism for the etching process. The oxidation 197 mechanisms might be a more valid explanation for our obser198 vations as metals are likely to be oxidized during metal evapora199 tion or as a result of exposure to oxygen during handling or in 200 the hydrocarbon contamination. It should be noted that 201 the above cited studies were performed at high temperatures $202\left(>650{ }^{\circ} \mathrm{C}\right)$, under gas flow on a substrate, whereas our experi203 ments are performed at room temperature under ultrahigh vacuum 204 conditions. However, although no heat was applied, the energy 205 transferred by the electron beam to the metal-graphene system 206 could be sufficient to activate the etching mechanism, bearing in 207 mind graphene's large heat conductivity; postscanning overviews 208 at lower magnification revealed that holes have also formed in the 209 proximity, that is, outside of consecutively e-beam-scanned areas. 210 Titanium reacts even more strongly with graphene, as also 211 predicted by DFT calculations, ${ }^{38}$ which is reflected in the large 212 binding energy, and thus affects the Ti mobility on graphene. 213 This is confirmed by the appearance of atomic-size aggregates, 214 rather than clusters of $\mathrm{Ti}$ on graphene (Figure $5 \mathrm{a}$ ). Ti has the 215 highest observed dispersion out of the metals studied here. For 216 this reason, $\mathrm{Ti}$ atoms do not need to be mobilized over larger 217 distances, and holes form already during the first scan. 218 Although, as in the case of the other metals, Ti is mainly 219 found on hydrocarbon chains, it sometimes resides on clean 220 graphene; this can be witnessed by the fact that etching does 221 not only occur on the border between clean graphene and 222 hydrocarbon deposits but also directly on the basal plane of 223 graphene (Figure 5b). Most recently, because of its thermal 224 stability, palladium has been used in graphene-based devices as 225 an electrical contact ${ }^{39}$ and for heterogeneous catalytic 226 applications. ${ }^{40} \mathrm{Pd}$ has been predicted by recent DFT calcula227 tions to form three-dimensional clusters on graphene. This is an 228 indication of its weak interaction with graphene, as also 229 predicted for many other transition metals. ${ }^{5}$ We have evidenced 230 that $\mathrm{Pd}$ appears in cluster form rather than highly dispersed like $231 \mathrm{Al}, \mathrm{Ti}$, and $\mathrm{Cr}$ (Figure 5c). Although the clustering behavior is 232 reminiscent of that of $\mathrm{Au}$ on graphene, in contrast to the latter, $233 \mathrm{Pd}$ does etch graphene (not shown).

234 Chromium is also found to be very reactive with graphene, and, 235 similar to $\mathrm{Ti}$, individual $\mathrm{Cr}$ adatoms have been observed on the 236 clean graphene surface. Etching is seen to commence directly in 237 the basal plane as well as at the border between clean graphene 238 and hydrocarbon contamination (Figure 5d). Coalescence of the 239 holes occurs during subsequent scans (Figure 5e).

240 In summary, we have shown how different metals interact 241 with suspended graphene. All studied metals favor sites on 242 hydrocarbon contamination rather than on the clean graphene

\author{
All of the metals studied herein \\ favor sites on hydrocarbon con- \\ tamination rather than on the \\ clean graphene surface and \\ present nonuniform distribu- \\ tions, which indicates a weak \\ interaction between the metal \\ and graphene.
}

surface and present nonuniform distributions, which indicates a 243 weak interaction between the metal and graphene. This behav- 244 ior is slightly altered with hydrogenation, and it would be 245 worthwhile to investigate other surface treatments, for example, 246 fluorination. High-temperature vacuum annealing is the only 247 way to get rid of the hydrocarbon contamination, which accom- 248 modates most of the metal nanoparticles; this is important, for 249 instance, for in situ observations of catalytic activities and of the 250 movement of metal nanoclusters on graphene. Graphene etch- 251 ing is observed for all metals considered in this study, except for 252 $\mathrm{Au}$. This unusual metal-mediated etching of graphene in a 253 STEM in ultrahigh vacuum at $60 \mathrm{kV}$ acceleration voltage sheds 254 new light on the metal-graphene interaction and could be ex- 255 ploited in controlled nanomanipulation and self-assembly pro- 256 cesses for future graphene-based devices.

\section{AUTHOR INFORMATION}

\section{Corresponding Author}

*E-mail: ursel.bangert@manchester.ac.uk.

\section{Notes}

The authors declare no competing financial interest.

\section{Biographies}

263

Recep Zan is currently studying for a Ph.D. in the School of Physics 264 under the supervision of U. Bangert and K. Novoselov at the 265 University of Manchester. He received his B.S. and M.Sc. degrees in 266 Physics from Cukurova University, Turkey. His Ph.D. project is based 267 on transmission electron microscopy of $2 \mathrm{D}$ materials, in particular, 268 their interaction with metals.

Ursel Bangert is a Reader in the School of Materials at The University 270 of Manchester. She obtained her Ph.D. from the University of 271 Cologne, Germany. She has contributed to the advancement and 272 exploration of electron microscopies/spectroscopies with ultrahigh spatial 273 resolution. Her research has centred around functional materials and, 274 more recently, nanostructured materials. www.manchester.ac.uk/research/ 275 ursel.bangert/

Quentin Ramasse is Scientific Director at the Deresbury SuperSTEM 277 Laboratories, the EPSRC National Facility for Aberration-Corrected 278 STEM. He obtained his Ph.D. from the University of Cambridge and 279 was previously a Staff Scientist at the National Center for Electron 280 Microscopy in Berkeley, California. www.superstem.ac.uk

Konstantin Novoselov is a Professor and Royal Society Research 282 Fellow in the School of Physics at The University of Manchester. He 283 has been awarded the Nobel Prize in Physics, 2010, "for 284 groundbreaking experiments regarding the two-dimensional material 285 
286 graphene". He obtained his Ph.D. from the University of Nijmegen, 287 The Netherlands. His research interests are mesoscopic systems and 288 nanostructures. www.Kostya.graphene.org

\section{ACKNOWLEDGMENTS}

290 This work is supported by EPSRC (U.K.).

\section{REFERENCES}

292 (1) Novoselov, K. S.; Geim, A. K.; Morozov, S. V.; Jiang, D.; Zhang, 293 Y.; Dubonos, S. V.; Grigorieva, I. V.; Firsov, A. A. Electric Field Effect 294 in Atomically Thin Carbon Films. Science 2004, 306, 666-669.

295 (2) Geim, A. K.; Novoselov, K. S. The rise of graphene. Nat. Mater. 296 2007, 6, 183-191.

297 (3) Pi, K.; McCreary, K. M.; Bao, W.; Han, W.; Chiang, Y. F.; Li, Y.; 298 Tsai, S. W.; Lau, C. N.; Kawakami, R. K. Electronic Doping and 299 Scattering by Transition Metals on Graphene. Phys. Rev. B 2009, 80, 300075406.

301 (4) Venugopal, A.; Colombo, L.; Vogel, E. M. Contact Resistance in 302 Few and Multilayer Graphene Devices. Appl. Phys. Lett. 2010, 96, 303013512.

304 (5) Chan, K. T.; Neaton, J. B.; Cohen, M. L. First-Principles Study of 305 Metal Adatom Adsorption on Graphene. Phys. Rev. B 2008, 77, 306235430 .

307 (6) Krasheninnikov, A. V.; Lehtinen, P. O.; Foster, A. S.; Pyykkö, P.; 308 Nieminen, R. M. Embedding Transition-Metal Atoms in Graphene: 309 Structure, Bonding, and Magnetism. Phys. Rev. Lett. 2009, 102, 310126807.

311 (7) Boukhvalov, D. W.; Katsnelson, M. I. Chemical Functionalization 312 of Graphene with Defects. Nano Lett. 2008, 8, 4373-4379.

313 (8) Cretu, O.; Krasheninnikov, A. V.; Rodriguez-Manzo, J. A.; Sun, 314 L.; Nieminen, R. M.; Banhart, F. Migration and Localization of Metal 315 Atoms on Strained Graphene. Phys. Rev. Lett. 2010, 105, 196102.

316 (9) Zhou, M.; Zhang, A.; Dai, Z.; Feng, Y. P.; Zhang, C. Strain317 Enhanced Stabilization and Catalytic Activity of Metal Nanoclusters 318 on Graphene. J. Phys. Chem. C 2010, 114, 16541-16546.

319 (10) Rodriguez-Manzo, J. A.; Cretu, O.; Banhart, F. Trapping of 320 Metal Atoms in Vacancies of Carbon Nanotubes and Graphene. ACS 321 Nano 2010, 4, 3422-3428.

322 (11) Kamat, P. V. Graphene-Based Nanoarchitectures. Anchoring 323 Semiconductor and Metal Nanoparticles on a Two-Dimensional 324 Carbon Support. J. Phys. Chem. Lett. 2009, 1, 520-527.

325 (12) Muszynski, R.; Seger, B.; Kamat, P. V. Decorating Graphene 326 Sheets with Gold Nanoparticles. J. Phys. Chem. C 2008, 112, 52633275266.

328 (13) Jasuja, K.; Linn, J.; Melton, S.; Berry, V. Microwave-Reduced 329 Uncapped Metal Nanoparticles on Graphene: Tuning Catalytic, 330 Electrical, and Raman Properties. J. Phys. Chem. Lett. 2010, 1, $331 \quad 1853-1860$.

332 (14) Pandey, P. A.; Bell, G. R.; Rourke, J. P.; Sanchez, A. M.; Elkin, 333 M. D.; Hickey, B. J.; Wilson, N. R. Physical Vapor Deposition of Metal 334 Nanoparticles on Chemically Modified Graphene: Observations on 335 Metal-Graphene Interactions. Small 2011, 7, 3202-3210.

336 (15) Kundu, P.; Nethravathi, C.; Deshpande, P. A.; Rajamathi, M.; 337 Madras, G.; Ravishankar, N. Ultrafast Microwave-Assisted Route to 338 Surfactant-Free Ultrafine Pt Nanoparticles on Graphene: Synergistic 339 Co-reduction Mechanism and High Catalytic Activity. Chem. Mater. $3402011,23,2772-2780$.

341 (16) Lee, Z.; Jeon, K.-J.; Dato, A.; Erni, R.; Richardson, T. J.; 342 Frenklach, M.; Radmilovic, V. Direct Imaging of Soft-Hard Interfaces 343 Enabled by Graphene. Nano Lett. 2009, 9, 3365-3369.

344 (17) Warner, J. H.; Rummeli, M. H.; Bachmatiuk, A.; Wilson, M.; 345 Buchner, B. Examining Co-Based Nanocrystals on Graphene Using 346 Low-Voltage Aberration-Corrected Transmission Electron Micros347 copy. ACS Nano 2009, 4, 470-476.

348 (18) Schedin, F.; Lidorikis, E.; Lombardo, A.; Kravets, V. G.; Geim, 349 A. K.; Grigorenko, A. N.; Novoselov, K. S.; Ferrari, A. C. Surface350 Enhanced Raman Spectroscopy of Graphene. ACS Nano 2010, 4, $3515617-5626$
(19) Lee, J.; Novoselov, K. S.; Shin, H. S. Interaction between Metal 352 and Graphene: Dependence on the Layer Number of Graphene. ACS 353 Nano 2011, 5, 608-612.

(20) N’Diaye, A. T.; Bleikamp, S.; Feibelman, P. J.; Michely, T. Two- 355 Dimensional Ir Cluster Lattice on a Graphene Moiré on $\operatorname{Ir}(111)$. Phys. 356 Rev. Lett. 2006, 97, 215501.

(21) Sicot, M.; Bouvron, S.; Zander, O.; Rudiger, U.; Dedkov, Y. S.; 358 Fonin, M. Nucleation and Growth of Nickel Nanoclusters on 359 Graphene Moire on $\mathrm{Rh}(111)$. Appl. Phys. Lett. 2010, 96, 093115-3. 360

(22) Zhou, Z.; Gao, F.; Goodman, D. W. Deposition of Metal 361 Clusters on Single-Layer Graphene/Ru(0001): Factors That Govern 362 Cluster Growth. Surf. Sci. 2010, 604, L31-L38.

(23) Biró, L. P.; Lambin, P. Nanopatterning of Graphene with 364 Crystallographic Orientation Control. Carbon 2010, 48, 2677-2689. 365

(24) Campos, L. C.; Manfrinato, V. R.; Sanchez-Yamagishi, J. D.; 366 Kong, J.; Jarillo-Herrero, P. Anisotropic Etching and Nanoribbon 367 Formation in Single-Layer Graphene. Nano Lett. 2009, 9, 2600-2604. 368

(25) Severin, N.; Kirstein, S.; Sokolov, I. M.; Rabe, J. P. Rapid Trench 369 Channeling of Graphenes with Catalytic Silver Nanoparticles. Nano 370 Lett. 2008, 9, 457-461.

(26) Ponomarenko, L. A.; Geim, A. K.; Zhukov, A. A.; Jalil, R; 372 Morozov, S. V.; Novoselov, K. S.; Grigorieva, I. V.; Hill, E. H.; Cheianov, 373 V. V.; Falko, V. I.; et al. Tunable Metal-Insulator Transition in Double- 374 Layer Graphene Heterostructures. Nat. Phys. 2011, 7, 958-961. 375

(27) Hong, W.; Bai, H.; Xu, Y.; Yao, Z.; Gu, Z.; Shi, G. Preparation of 376 Gold Nanoparticle/Graphene Composites with Controlled Weight 377 Contents and Their Application in Biosensors. J. Phys. Chem. C 2010, 378 $114,1822-1826$

(28) Seger, B.; Kamat, P. V. Electrocatalytically Active Graphene- 380 Platinum Nanocomposites. Role of 2-D Carbon Support in PEM Fuel 381 Cells. J. Phys. Chem. C 2009, 113, 7990-7995.

382

(29) Chen, D.; Ji, G.; Ma, Y.; Lee, J. Y.; Lu, J. Graphene-Encapsulated 383 Hollow $\mathrm{Fe}_{3} \mathrm{O}_{4}$ Nanoparticle Aggregates As a High-Performance Anode 384 Material for Lithium Ion Batteries. ACS Appl. Mater. Interfaces 2011, 3, 385 $3078-3083$

(30) Li, X.; Cai, W.; An, J.; Kim, S.; Nah, J.; Yang, D.; Piner, R.; 387 Velamakanni, A.; Jung, I.; Tutuc, E.; et al. Large-Area Synthesis of 388 High-Quality and Uniform Graphene Films on Copper Foils. Science 389 2009, 324, 1312-1314.

(31) Meyer, J. C.; Geim, A. K.; Katsnelson, M. I.; Novoselov, K. S.; 391 Obergfell, D.; Roth, S.; Girit, C.; Zettl, A. On the Roughness of Single- 392 and Bi-layer Graphene Membranes. Solid State Commun. 2007, 143, 393 101-109.

394

(32) Gan, Y.; Sun, L.; Banhart, F. One- and Two-Dimensional 395 Diffusion of Metal Atoms in Graphene. Small 2008, 4, 587-591. 396

(33) Zan, R.; Bangert, U.; Ramasse, Q.; Novoselov, K. S. Metal- 397 Graphene Interaction Studied via Atomic Resolution Scanning 398 Transmission Electron Microscopy. Nano Lett. 2011, 11, 1087-1092. 399

(34) Elias, D. C.; Nair, R. R.; Mohiuddin, T. M. G.; Morozov, S. V.; 400 Blake, P.; Halsall, M. P.; Ferrari, A. C.; Boukhvalov, D. W.; Katsnelson, 401 M. I.; Geim, A. K.; et al. Control of Graphene's Properties by 402 Reversible Hydrogenation: Evidence for Graphane. Science 2009, 323, 403 610-613.

(35) Zan, R.; Bangert, U.; Ramasse, Q.; Novoselov, K. S. Evolution of 405 Gold Nanostructures on Graphene. Small 2011, 7, 2868-2872. 406

(36) Egerton, R. F.; Wang, F.; Crozier, P. A. Beam-Induced Damage 407 to Thin Specimens in an Intense Electron Probe. Microsc. Microanal. 408 2006, 12, 65-71.

(37) Boukhvalov, D. W.; Katsnelson, M. I. Destruction of Graphene 410 by Metal Adatoms. Appl. Phys. Lett. 2009, 95, 023109.

(38) Giovannetti, G.; Khomyakov, P. A.; Brocks, G.; Karpan, V. M.; 412 van den Brink, J.; Kelly, P. J. Doping Graphene with Metal Contacts. 413 Phys. Rev. Lett. 2008, 101, 026803.

(39) Xia, F.; Perebeinos, V.; Lin, Y.-m.; Wu, Y.; Avouris, P. The 415 Origins and Limits of Metal-Graphene Junction Resistance. Nat Nano 416 2011, 6, 179-184.

(40) Jin, Z.; Nackashi, D.; Lu, W.; Kittrell, C.; Tour, J. M. 418 Decoration, Migration, and Aggregation of Palladium Nanoparticles on 419 Graphene Sheets. Chem. Mater. 2010, 22, 5695-5699. 